\title{
Metabolic Syndrome With Aortic Arterial Stiffness And First Hospitalization Or Mortality In Coronary Artery Disease Patients
}

This article was published in the following Dove Press journal: Diabetes, Metabolic Syndrome and Obesity: Targets and Therapy

\author{
Yen-Chih Chen $\mathbb{D}^{1, *}$ \\ Bang-Gee Hsu (iD) $2,3, *$ \\ Ji-Hung Wang $\mathbb{D}^{3,4}$ \\ Chung-Jen Lee $\mathbb{D}^{5}$ \\ Jen-Pi Tsai ${ }^{3,6}$
}

'Department of Psychiatry, Hualien Hospital, Ministry of Health and Welfare, Hualien, Taiwan; ${ }^{2}$ Division of Nephrology, Hualien Tzu Chi Hospital, Buddhist Tzu Chi Medical Foundation, Hualien, Taiwan; ${ }^{3}$ School of Medicine, Tzu Chi University, Hualien, Taiwan; ${ }^{4}$ Division of Cardiology, Hualien Tzu Chi Hospital, Buddhist Tzu Chi Medical Foundation, Hualien, Taiwan: ${ }^{5}$ Department of Nursing, Tzu Chi University of Science and Technology, Hualien, Taiwan; ${ }^{6}$ Division of Nephrology, Department of Internal Medicine, Dalin Tzu Chi Hospital, Buddhist Tzu Chi Medical Foundation, Chiayi, Taiwan

*These authors contributed equally to this work
Correspondence: Ji-Hung Wang Division of Cardiology, Hualien Tzu Chi Hospital, Buddhist Tzu Chi Medical Foundation, No. 707, Section 3, ChungYang Road, Hualien 97010, Taiwan Email abanggeelily@gmail.com

Jen-Pi Tsai

Division of Nephrology, Department of Internal Medicine, Dalin Tzu Chi Hospital, Buddhist Tzu Chi Medical Foundation,

No. 2, Min-Sheng Road, Dalin Town,

Chiayi 62247, Taiwan

Tel +88652648000

Fax +88652648006

Email tsaininimdI49I@outlook.com
Purpose: Metabolic syndrome (MetS) and aortic arterial stiffness (AS) are risk factors for future cardiovascular events. We evaluated their roles in first hospitalization or all-cause mortality prediction in coronary artery disease (CAD) patients.

Patients and methods: From January to December 2012, 115 CAD patients were enrolled from a single center and followed up for 5.5 years. The composite endpoint included hospitalization for unstable angina, myocardial infarction, revascularization, or heart failure and all-cause mortality. Patients with carotid-femoral pulse wave velocity $>10 \mathrm{~m} / \mathrm{s}$ (measured using applanation tonometry) constituted the high AS group.

Results: During a median 54-month follow-up, there were $43(37.4 \%)$ and $11(9.6 \%)$ hospitalization and mortality events, respectively. Overall, 41 (35.7\%) and $70(60.9 \%)$ patients were diagnosed with AS and MetS, respectively. CAD patients with high AS had higher diabetes and MetS percentages, were older, and had higher waist circumference and systolic blood pressure (SBP) but lower glomerular filtration rate than those with low AS. Multivariate logistic regression analysis revealed old age $(P<0.001)$, diabetes $(P=0.003)$, and high waist circumference $(P=0.044)$ and SBP $(P=0.007)$ as independent predictors of AS in CAD patients. Kaplan-Meier analysis showed that CAD patients with concurrent MetS and high AS had a higher risk for hospitalization (log rank test, $P=0.005$ ) or developing all-cause mortality (log rank test, $P=0.002$ ). Compared with CAD patients without MetS or AS, composite outcome development risk in those with both the conditions was 10.2-fold higher $(P<0.001)$; this risk was 6.54-fold higher in those with AS alone $(P=0.007)$.

Conclusion: In CAD patients, age, diabetes, and high waist circumference and SBP are the independent predictors of AS. Additionally, CAD patients with AS with and without MetS have a high first hospitalization or all-cause mortality development risk.

Keywords: aortic arterial stiffness, carotid-femoral pulse wave velocity, coronary artery disease, hospitalization, metabolic syndrome, mortality

\section{Introduction}

Metabolic syndrome (MetS) is defined as central obesity accompanied by features such as hypertriglyceridemia, low high-density lipoprotein cholesterol (HDL-C) levels, hyperglycemia, and hypertension (HTN); this condition adversely affects cardiovascular $(\mathrm{CV})$ outcomes. ${ }^{1,2}$ Evidence had shown that there is correlation between adverse $\mathrm{CV}$ events such as ischemic stroke and immune-inflammatory activation. $^{3-5}$ Also, the features characterizing MetS are reportedly associated with a proinflammatory state through a combination of atherogenic dyslipidemia, dysglycemia, and a prothrombotic state. ${ }^{1,2}$ Earlier longitudinal studies have shown 
that men with at least three features of MetS have an increased risk of coronary artery disease (CAD) and diabetes mellitus (DM) development. ${ }^{6,7}$ Evidence has shown that compared with patients without MetS, those with MetS have a significantly increased adjusted risk of mortality from CAD and cardiovascular disease (CVD) and all-cause mortality. ${ }^{8}$

Arterial stiffness (AS) due to loss of arterial compliance caused by phenomena such as elastin fiber and collagen deregulation, cellular element re-organization, and low-grade inflammation results in a more rapid travel time and hence a high pulse wave velocity (PWV). Carotidfemoral pulse wave velocity (cfPWV) is a non-invasive and simple clinical method to evaluate AS, and AS is an emerging CVD marker. ${ }^{9}$ Studies have suggested a relationship of increased PWV with coronary artery calcium score and subclinical $\mathrm{CAD}$ and suggested that $\mathrm{AS}$ could be a predictor for CVD. ${ }^{10,11}$ Moreover, a meta-analysis has shown that AS, represented as aortic PWV, is a strong predictor of future $\mathrm{CV}$ events and all-cause mortality; additionally, the study has reported that AS's predictive ability is higher in subjects with a higher baseline CV risk. $^{9}$

In a study conducted in Chinese HTN patients, increasing levels of cfPWV with an increasing number of MetS components independent of age or gender were observed. ${ }^{12}$ In an earlier cross-sectional study on DM patients, AS, defined by cfPWV, positively correlated with MetS. ${ }^{13}$ Moreover, MetS components including obesity, HTN, hypertriglyceridemia, and low HDL-C levels have been shown to be significantly associated with higher annual medical costs. ${ }^{14}$ However, while increased PWV has been shown to predict a greater risk of $\mathrm{CV}$ morbidity and mortality in HTN and DM patients, ${ }^{15}$ the association between AS and CAD patients with or without MetS remains unclear. To overcome this limitation, the present study aimed to examine the risk factors for AS in CAD patients and the influences of MetS and AS on first hospitalization or all-cause mortality in these patients.

\section{Materials And Methods}

\section{Patients}

In total, $115 \mathrm{CAD}$ participants were enrolled in this longitudinal study; the participants were found to have a history of CAD between January and December 2012 at the Buddhist Tzu Chi General Hospital, Hualien, Taiwan. In this study, CAD was defined as any segment of coronary arteries with $>50 \%$ stenosis observed on angiography following medical record review. Blood pressure (BP) for all participants was measured with automatic sphygmomanometer by trained staff in the morning after $>10$-min rest. Systolic BP (SBP) and diastolic BP (DBP) were recorded three times at 5-min intervals; the measurements were averaged for analysis. Patients with an SBP $\geq 140 \mathrm{mmHg}$ and/or DBP $\geq 90 \mathrm{mmHg}$ or those who had received any anti-HTN medication over the past 2 weeks were defined as having HTN. Patients were diagnosed with DM if either their fasting plasma glucose levels were $\geq 126 \mathrm{mg} / \mathrm{dL}$ or they were using oral hypoglycemic medications or insulin. Exclusion criteria were as follows: presence of acute inflammatory diseases, acute $\mathrm{CAD}$, pulmonary edema at the time of blood sampling, or refusal to provide informed consent for the study. An informed consent was obtained from all patients prior to study participation. The study was approved by The Protection of the Human Subjects Institutional Review Board of Tzu-Chi University and Hospital.

\section{Anthropometric Analysis}

As done in our previous studies, body weight and body height were measured and waist circumference was measured at the midpoint between the lowest ribs and the iliac crest with the participants in light clothing without shoes. Body mass index (BMI) was calculated as the weight in kilograms divided by the height in meters squared. ${ }^{16-18}$

\section{Biochemical Examinations}

After 8-h overnight fasting, blood samples (approximately $5 \mathrm{~mL}$ ) of all participants were collected and sent for examination. Laboratory tests performed were as follows: serum levels of blood urea nitrogen (BUN), creatinine (Cre), fasting glucose, total cholesterol, triglyceride (TG), HDL-C, lowdensity lipoprotein cholesterol, total calcium, and phosphorus. An autoanalyzer (COBAS Integra 800, Roche Diagnostics, Basel, Switzerland) was used for performing the tests. Serum levels of intact parathyroid hormone (iPTH) (Diagnostic Systems Laboratories, Texas, USA) were measured using a commercially available enzymelinked immunosorbent assay. Finally, the estimated glomerular filtration rate (eGFR) was calculated using the Chronic Kidney Disease Epidemiology Collaboration equation. ${ }^{16-18}$

\section{Metabolic Syndrome}

MetS was defined as per the International Diabetes Federation definition, which includes central obesity with a waist circumference of $\geq 90 \mathrm{~cm}$ (men) or $\geq 80 \mathrm{~cm}$ 
(women) (Chinese criteria) and meeting at least two of the following criteria: fasting glucose levels $\geq 100 \mathrm{mg} / \mathrm{dL}, \mathrm{TG}$ levels $\geq 150 \mathrm{mg} / \mathrm{dL}$, HDL-C levels of $<40 \mathrm{mg} / \mathrm{dL}$ (men) or $<50 \mathrm{mg} / \mathrm{dL}$ (women), or $\mathrm{BP} \geq 130 / 85 \mathrm{mmHg}$. ${ }^{19}$ Serum insulin levels were measured using the microparticle enzyme immunosorbent assay method with an autoanalyzer (Abbott Laboratories, Abbott Park, IL, USA). Insulin resistance was calculated using a homeostasis model assessment of insulin resistance (HOMA-IR) as follows: HOMA-IR = fasting plasma glucose $(\mathrm{mg} / \mathrm{dL}) \times$ fasting serum insulin $(\mu \mathrm{U} / \mathrm{mL}) / 405 .^{17}$

\section{Carotid-Femoral Pulse Wave Velocity Measurements}

After at least 10-min rest in a quiet and temperature-controlled room in the morning, cfPWV measurements were performed once for all patients by one trained staff using pressure applanation tonometry (SphygmoCor system, AtCor Medical, Australia) in the supine position, as done in previous studies. ${ }^{16,18}$ Pulse wave recordings were performed electrocardiogram (ECG)-gated simultaneously with an R-timing reference and consecutively at the carotid and femoral arteries (the carotid-femoral segment). Integral software was used to process each pulse wave and ECG data set to calculate the mean time difference between $R$-wave and pulse wave on a beat-to-beat basis, with an average of 10 consecutive cardiac cycles. Subsequently, cfPWV was calculated using distance and the mean time difference between the two recorded points. As previously defined, patients with cfPWV values $>10$ $\mathrm{m} / \mathrm{s}$ were defined as the high AS group. ${ }^{15,16,18}$

\section{Follow-Up And Endpoints}

Patients' conditions were traced by regular outpatient services or via telephone (in cases of physical immobility) until June 30, 2017. The endpoints were defined as follows: first hospitalization for unstable angina, myocardial infarction, revascularization, and heart failure or all-cause mortality. The event-free survival was defined as the interval between the date of cfPWV evaluation and the date of occurrence of endpoints or the end of follow-up.

\section{Statistical Analysis}

Kolmogorov-Smirnov test was used to analyze continuous variables for normal distribution. Normally distributed variables were expressed as means \pm standard deviations, and Student's $t$-test (two-tailed) was used to compare the patients. Non-normally distributed variables were expressed as medians and interquartile ranges (IQRs), and patients were compared using Mann-Whitney $U$-test. Categorical variables were expressed as patient numbers and percentages and were analyzed using chi-square test. For further analysis, patients were sub-grouped into four groups as follows: no MetS + no AS, MetS + no AS, no MetS + AS, and MetS + AS. Comparisons among the groups were performed using Kruskal-Wallis analysis or one-way analysis of variance for parameters that presented without or with normal distribution, respectively. Multivariate logistic regression analysis was used to analyze variables associated with the presence of high AS in CAD patients. Kaplan-Meier survival curves with a log rank test were used for comparing the cumulative proportion of CAD patients free from first hospitalization or mortality among these four groups. Multivariate Cox regression models were used to analyze variables associated with the risk of first hospitalization. Data were analyzed using SPSS for Windows (version 19.0; SPSS Inc., Chicago, IL, USA). $P$-values $<0.05$ were considered significant.

\section{Results}

The baseline characteristics of the $115 \mathrm{CAD}$ patients, divided into low and high $(\mathrm{n}=41,35.7 \%)$ AS, are described in Table 1. Among the 115 patients, 54 (48.7\%), 92 $(80.0 \%)$, and $70(60.9 \%)$ patients were defined as having DM, HTN, and MetS, respectively.

On sub-grouping the CAD patients, 34 (29.5\%), 11 (9.6\%), 40 (34.8\%), and 30 (26.1\%) patients were categorized into the no MetS + no AS, MetS + no AS, no MetS + AS, and MetS + AS groups, respectively (Table 2). Interestingly, significant differences were identified among these groups in age; waist circumference; BMI; cfPWV values; SBP; eGFR; TG, HDL-C, fasting glucose, BUN, total calcium, phosphorous, calcium-phosphorous product, and insulin levels; and HOMA-IR. After adjusting the risk factors including age, waist circumference, SBP, eGFR, DM, and MetS, old age $(P<0.001)$, DM $(P=0.003)$, high waist circumference $(P=0.044)$, and SBP $(P=0.007)$ were identified as the independent predictors of AS in CAD patients (Table 3 ).

During a median 54-month follow-up, 43 (37.4\%) first hospitalization and $11(9.6 \%)$ mortality events were observed. Kaplan-Meier analysis showed that CAD patients with both MetS and AS had the worst free-survival from first hospitalization (log rank test, $P=0.005$ ) (Figure 1A) or allcause mortality (log rank test, $P=0.002$ ) (Figure 1B). For 
Table I Clinical Variables Of The II 5 Coronary Artery Disease Patients With Or Without Aortic Arterial Stiffness

\begin{tabular}{|c|c|c|c|c|}
\hline Variables & $\begin{array}{l}\text { All Participants } \\
(\mathrm{n}=1 \mid 5)\end{array}$ & $\begin{array}{l}\text { No Arterial Stiffness } \\
\text { Group }(n=74)\end{array}$ & $\begin{array}{l}\text { High Arterial Stiffness } \\
\text { Group }(n=4 I)\end{array}$ & $P$ value \\
\hline Age (years) & $65.61 \pm 8.97$ & $63.38 \pm 8.57$ & $69.63 \pm 8.32$ & $<0.001 *$ \\
\hline Height $(\mathrm{cm})$ & $|6| .2 \mid \pm 8.08$ & $|6| .53 \pm 7.4 \mid$ & $160.63 \pm 9.23$ & 0.572 \\
\hline Body weight (kg) & $68.31 \pm 11.84$ & $67.41 \pm 11.63$ & $69.94 \pm 12.17$ & 0.273 \\
\hline Waist circumference $(\mathrm{cm})$ & $93.08 \pm 9.75$ & $91.50 \pm 9.41$ & $95.93 \pm 9.82$ & $0.019 *$ \\
\hline Body mass index $\left(\mathrm{kg} / \mathrm{m}^{2}\right)$ & $26.19 \pm 3.45$ & $25.73 \pm 3.34$ & $27.03 \pm 3.53$ & 0.053 \\
\hline $\operatorname{cfPWV}(\mathrm{m} / \mathrm{s})$ & $9.52 \pm 2.60$ & $8.11 \pm 1.59$ & $12.06 \pm 2.09$ & $<0.00 I^{*}$ \\
\hline Systolic blood pressure $(\mathrm{mmHg})$ & $132.04 \pm 18.14$ & $127.72 \pm 15.50$ & $139.85 \pm 20.05$ & $<0.001 *$ \\
\hline Diastolic blood pressure $(\mathrm{mmHg})$ & $72.55 \pm 10.28$ & $71.74 \pm 10.32$ & $74.00 \pm 10.18$ & 0.261 \\
\hline Total cholesterol (mg/dL) & $165.67 \pm 35.19$ & $137.32 \pm 35.87$ & $162.68 \pm 34.17$ & 0.501 \\
\hline Triglycerides (mg/dL) & $121.00(90.00-166.00)$ & $115.00(89.00-184.25)$ & $127.00(90.50-152.50)$ & 0.829 \\
\hline $\mathrm{HDL}-\mathrm{C}(\mathrm{mg} / \mathrm{dL})$ & $44.92 \pm 11.95$ & $46.41 \pm 11.79$ & $42.24 \pm 11.92$ & 0.074 \\
\hline LDL-C (mg/dL) & $95.83 \pm 26.53$ & $96.08 \pm 25.31$ & $95.37 \pm 28.93$ & 0.891 \\
\hline Fasting glucose (mg/dL) & $111.00(96.00-137.00)$ & $106.00(96.75-129.50)$ & $118.00(95.00-188.50)$ & 0.140 \\
\hline Blood urea nitrogen $(\mathrm{mg} / \mathrm{dL})$ & $16.00(13.00-19.00)$ & $16.00(13.00-18.00)$ & $17.00(\mid 3.00-21.50)$ & 0.136 \\
\hline Creatinine $(\mathrm{mg} / \mathrm{dL})$ & $1.10(0.90-1.30)$ & $1.00(0.90-1.20)$ & $1.20(0.90-1.45)$ & 0.054 \\
\hline Glomerular filtration rate $(\mathrm{mL} / \mathrm{min})$ & $68.73 \pm 19.43$ & $73.32 \pm 17.35$ & $62.26 \pm 21.45$ & $0.007^{*}$ \\
\hline Total calcium (mg/dL) & $9.12 \pm 0.35$ & $9.14 \pm 0.36$ & $9.09 \pm 0.35$ & 0.503 \\
\hline Phosphorus (mg/dL) & $3.52 \pm 0.54$ & $3.53 \pm 0.56$ & $3.5 \mathrm{I} \pm 0.5 \mathrm{I}$ & 0.804 \\
\hline Calcium-phosphorous product $\left(\mathrm{mg}^{2} / \mathrm{dL}^{2}\right)$ & $32.16 \pm 5.28$ & $32.32 \pm 5.55$ & $31.89 \pm 4.82$ & 0.678 \\
\hline Intact parathyroid hormone $(\mathrm{pg} / \mathrm{mL})$ & $52.87 \pm 26.81$ & $52.73 \pm 29.41$ & $53.10 \pm 21.70$ & 0.944 \\
\hline Insulin (ulU/mL) & $11.90(7.18-21.11)$ & II.06 (6.69-19.85) & $13.02(8.5 \mid-23.12)$ & 0.434 \\
\hline HOMA-IR & $3.67(2.11-7.27)$ & $3.47(1.81-7.06)$ & $4.00(2.73-7.33)$ & 0.250 \\
\hline Female (\%) & $28(24.3)$ & $20(27.0)$ & $8(19.5)$ & 0.368 \\
\hline Diabetes (\%) & $56(48.7)$ & $27(36.5)$ & $29(70.7)$ & $<0.00 I^{*}$ \\
\hline Hypertension (\%) & $92(80.0)$ & $56(75.7)$ & $36(87.8)$ & 0.119 \\
\hline Metabolic syndrome & $70(60.9)$ & $40(54.1)$ & $30(73.2)$ & $0.044^{*}$ \\
\hline
\end{tabular}

Notes: Values for continuous variables given as means \pm standard deviation and compared by Student's $t$-test; variables not normally distributed given as medians and interquartile range and compared by Mann-Whitney $U$-test; values are presented as number (\%), and analysis was performed using the chi-square test. $* P<0.05$ was considered statistically significant.

Abbreviations: cfPWV, carotid-femoral pulse wave velocity; HDL-C, high-density lipoprotein cholesterol; LDL-C, low-density lipoprotein cholesterol; HOMA-IR, homeostasis model assessment of insulin resistance; $A R B$, angiotensin-receptor blocker; $A C E$, angiotensin-converting enzyme; $C C B$, calcium-channel blocker.

further analyzing risk factors for developing endpoints using multivariate Cox regression analysis, variables including age, waist circumference, and BMI were adjusted in model 1. Additionally, variables in model 1 as well as SBP; TG, HDL-C, fasting glucose, and insulin levels; and HOMA-IR were adjusted in model 2. Finally, variables in model 2 as well as BUN, Cre, total calcium, phosphorous, and calciumphosphorous product levels and eGFR were adjusted in model 3 (Table 4). The results showed that compared with CAD patients without MetS or AS, those with both MetS and AS had a 7.38 -fold $(P<0.001), 8.23$-fold $(P<0.001)$, and 10.20-fold $(P<0.001)$ increased risk for developing endpoints as per Models 1, 2, and 3, respectively; furthermore, CAD patients with only AS had a 3.86-fold $(P=0.032), 5.48$ fold $(P=0.010)$, and 6.54-fold $(P=0.007)$ increased risk for developing endpoints as per Models 1,2 , and 3, respectively. No significant increase in the risk for developing endpoints was observed in CAD patients with MetS only compared with those without both MetS and AS.

\section{Discussion}

The present study demonstrated that old age, DM, a high waist circumference, and SBP were the predictors of high AS in CAD patients. Additionally, CAD patients with or without MetS but with high AS had a significantly higher risk for first hospitalization and all-cause mortality events than those without MetS and high AS.

Classic CV risk factors such as DM, dyslipidemia, elevated BMI, MetS, and smoking have been implicated in accelerating AS. ${ }^{10,12,13,20-22}$ Aging is characterized by dysregulated remodeling of elastin and collagen, causing loss of arterial elasticity and thickening of the vascular wall and increased arterial stiffening. ${ }^{21}$ Evidence has shown that AS measured using PWV has a significantly 


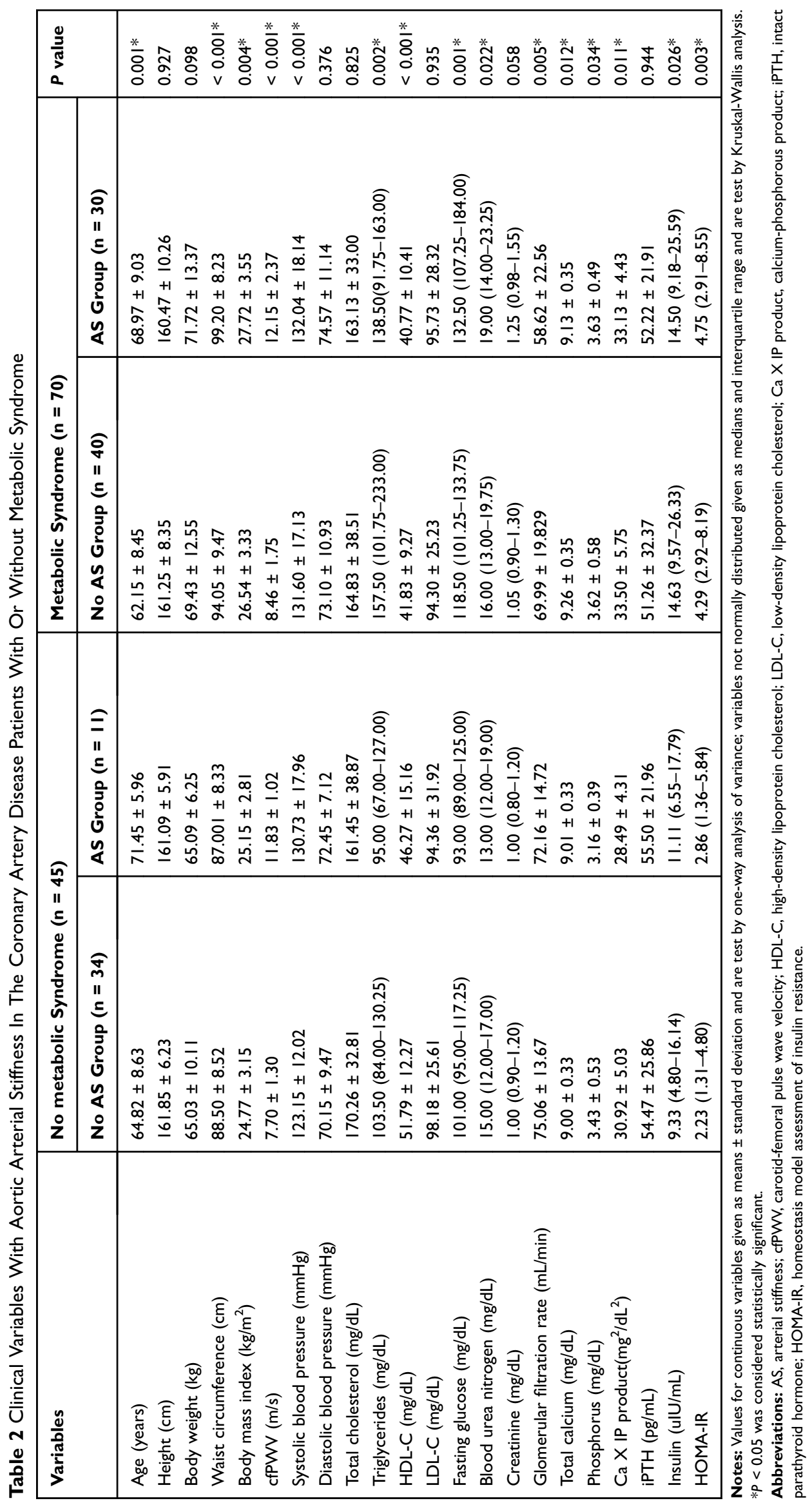


Table 3 Multivariate Logistic Regression Analysis Of The Factors Correlated With Aortic Stiffness Among The II5 Patients With Coronary Artery Disease

\begin{tabular}{|l|l|l|l|}
\hline Variables & $\begin{array}{l}\text { Odds } \\
\text { Ratio }\end{array}$ & $\begin{array}{l}\text { 95\% } \\
\text { Confidence } \\
\text { Interval }\end{array}$ & P value \\
\hline Age, I year & 1.13 & $1.06-1.21$ & $<0.00 I^{*}$ \\
Diabetes, present & 4.76 & $1.68-13.47$ & $0.003^{*}$ \\
Waist circumference, I cm & 1.06 & $1.00-1.11$ & $0.044^{*}$ \\
Systolic blood pressure, I & 1.04 & $1.01-1.08$ & $0.007^{*}$ \\
$\mathrm{mmHg}$ & & & \\
\hline
\end{tabular}

Notes: Analysis of data was done using the multivariate logistic regression analysis (adapted factors were diabetes, age, waist circumference, systolic blood pressure, glomerular filtration rate and metabolic syndrome). $* p<0.05$ was considered statistically significant.

positive correlation with patients' age in the presence of occult CAD, MetS, DM, or HTN. ${ }^{10,12,13,20}$ AS and HTN are pathophysiologically correlated, as increased AS reduces the lumen diameter and leads to a premature return of the reflected wave in late systole, resulting in increased pulse pressure and SBP and decreased DBP. ${ }^{20}$ Studies have shown a positive correlation of cfPWV with SBP as well as with MetS and increased waist circumference in HTN and DM patients. ${ }^{13,23}$ Moreover, a systemic review has reported that other than classic risk factors such as gender, dyslipidemia, smoking, and BMI, arterial aging and BP elevation are independently associated with $\mathrm{cfPWV}^{24}$ Schram et al have found that patients with impaired glucose metabolism and DM have lesser total

(A)

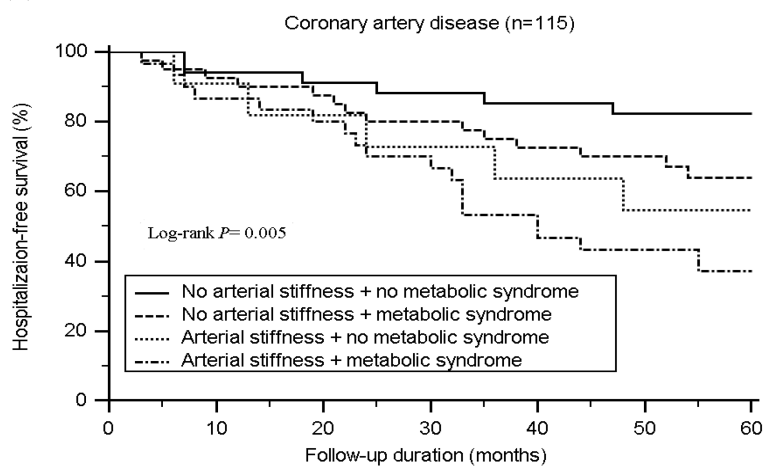

\begin{tabular}{llllllll} 
Months & 0 & 10 & 20 & 30 & 40 & 50 & 60 \\
\hline Number at risk & & & & & & & \\
\hline No AS + No & 34 & 32 & 31 & 30 & 29 & 28 & 0 \\
MS & & & & & & & \\
No AS + MS & 40 & 37 & 35 & 32 & 29 & 25 & 0 \\
AS + No MS & 11 & 10 & 9 & 8 & 7 & 6 & 0 \\
AS + MS & 30 & 26 & 24 & 20 & 14 & 12 & 0 \\
\hline
\end{tabular}

systemic arterial compliance, greater aortic augmentation index, and a lesser carotid-femoral transit time, which indicates an increased central AS compared with that in patients with normal glucose metabolism. ${ }^{25}$ Additionally, chronic DM has been found to be independently associated with increased cfPWV and macrovascular event risk after adjusting confounders. ${ }^{26}$ In a longitudinal study, adiposity was found to manifest as BMI and waist circumference and was positively correlated with cfPWV change, indicating that adiposity was also a predictor of $\mathrm{AS}^{27}$ In line with these reports, our study revealed that CAD patients with high AS with or without MetS, were significantly older, had higher percentages of DM comorbidity with higher fasting blood glucose levels, higher serum insulin levels and HOMA-IR, and a higher waist circumference and SBP than CAD patients with low AS without MetS. Furthermore, old age, DM, waist circumference, and SBP were found to be the possible risk factors for developing high AS after adjusting for covariates in CAD patients.

When evaluating eGFR $>60 \mathrm{~mL} / \mathrm{min} / 1.73 \mathrm{~m}^{2}$, Yan et al found that women with eGFR $<60 \mathrm{~mL} / \mathrm{min} / 1.73 \mathrm{~m}^{2}$ had higher PWV and that there was a negative association between eGFR and PWV. ${ }^{28}$ Two studies have been performed to examine the association between AS and renal function: Ilyas et al have found that CAD patients without a known renal disease exhibit a negative association between PWV and eGFR; Ford et al have found that in patients with more advanced chronic kidney disease (CKD) and an eGFR $15-59 \mathrm{~mL} / \mathrm{min} / 1.73 \mathrm{~m}^{2}$,

(B)

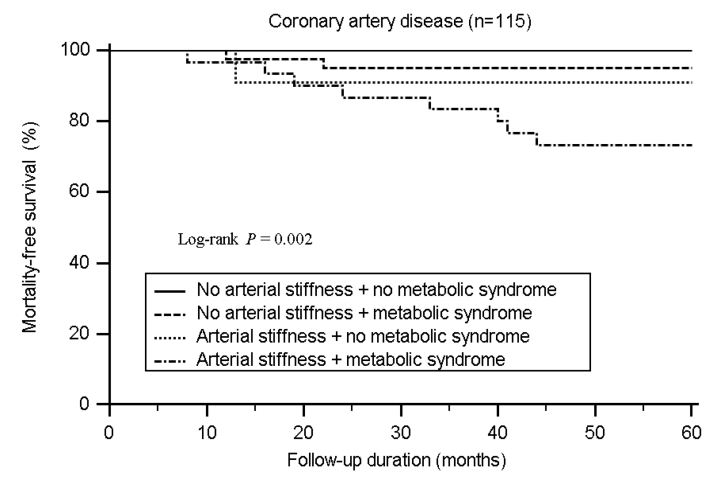

\begin{tabular}{lccccccc} 
Months & 0 & 10 & 20 & 30 & 40 & 50 & 60 \\
\hline Number at risk & & & & & & & \\
\hline No AS + No & 34 & 34 & 34 & 34 & 34 & 34 & 0 \\
MS & & & & & & & \\
No AS + MS & 40 & 40 & 39 & 38 & 38 & 35 & 0 \\
AS + No MS & 11 & 11 & 10 & 10 & 10 & 10 & 0 \\
AS + MS & 30 & 29 & 27 & 26 & 24 & 21 & 0
\end{tabular}

Figure I Kaplan-Meier analysis according to aortic arterial stiffness (AS) with or without metabolic syndrome (MS) for (A) first hospitalization (B) mortality in patients with coronary artery disease. 
Table 4 Adjusted Hazard Ratios For A Composite End-Point Consisting Of First Hospitalization Based On A Multivariate Cox Regression Model For Aortic Arterial Stiffness In The 115 Coronary Artery Disease Patients With Or Without Metabolic Syndrome

\begin{tabular}{|c|c|c|c|c|c|c|}
\hline & \multicolumn{2}{|l|}{ Model I } & \multicolumn{2}{|l|}{ Model 2} & \multicolumn{2}{|l|}{ Model 3} \\
\hline & Hazard Ratio $(95 \% \mathrm{Cl})$ & $P$ value & Hazard Ratio $(95 \% \mathrm{Cl})$ & $P$ value & Hazard Ratio (95\% Cl) & $P$ value \\
\hline No $A S$ and no MS & Reference & & Reference & & Reference & \\
\hline No $A S$ and $M S$ & $2.48(0.93-6.60)$ & 0.069 & $2.17(0.75-6.28)$ & 0.151 & $2.38(0.77-7.38)$ & 0.134 \\
\hline AS and no MS & $3.86(1.12-13.28)$ & $0.032 *$ & $5.48(1.50-20.05)$ & $0.010 *$ & $6.54(1.67-25.68)$ & $0.007^{*}$ \\
\hline AS and MS & $7.38(2.6 \mathrm{I}-20.83)$ & $<0.001^{*}$ & $8.23(2.46-27.86)$ & $<0.001 *$ & $10.20(2.77-37.53)$ & $<0.00 I^{*}$ \\
\hline
\end{tabular}

Notes: Model I is adjusted for age, waist circumference, and body mass index. Model 2 is adjusted for the model I variables and for systolic blood pressure, triglycerides, high-density lipoprotein cholesterol, fasting glucose, insulin, and homeostasis model assessment of insulin resistance. Model 3 is adjusted for the model 2 variables and for blood urea nitrogen, creatinine, glomerular filtration rate, total calcium, phosphorus, and calcium-phosphorous product. $* P<0.05$ was considered statistically significant. Abbreviations: AS, arterial stiffness; $\mathrm{MS}$, metabolic syndrome; $\mathrm{Cl}$, confidence interval.

AS is independently associated with a decline in renal function. ${ }^{9,29,30}$ Furthermore, in patients with advanced CKD, increased severity of AS is an independent predictor of $\mathrm{CV}$ events and mortality, with AS improving the risk prediction of these events. ${ }^{31,32}$ In this study, renal function did not appear as a risk factor for AS development after adjusting for covariates; however, still it was found that CAD patients with high AS with or without MetS have lower renal function. Together with the previously reported studies, we believe that renal function decline plays a role in AS development.

MetS, defined as central obesity along with associated factors such as dyslipidemia, hyperglycemia, and HTN, together with DM and chronic inflammation has been implicated in $\mathrm{CV}$ complications in urban population over 40 years old and in AS development. ${ }^{21,22}$ Evidence has shown that MetS and its components (ie, fasting glucose, BP, and waist circumference) are associated with $\mathrm{PWV}^{33}$ Yokoyama et al have found that as the number of MetS components increase, PWV significantly increases in patients with DM without CAD or renal diseases. ${ }^{34}$ Our results showed that CAD patients with MetS and AS had a higher waist circumference, SBP, hypertriglyceridemia, fasting glucose levels, cfPWV, and lower HDL-C levels than those without MetS and AS. Taken together, we believe that MetS plays a role in increased AS in CAD patients.

Central AS associated with increased systolic load and decreased myocardial perfusion pressure is an independent predictor of CV morbidity and mortality. ${ }^{29,31}$ Ilyas et al have found that in patients who have recently undergone coronary angiography, increased PWV is associated with future hospitalization due to CVD and all-cause mortality. ${ }^{29}$ Other longitudinal studies performed in CKD patients have shown AS, measured using PWV, as a significant predictor for future worse $\mathrm{CV}$ outcomes independent of age, gender, $\mathrm{BP}$, DM, or past CVD. ${ }^{31,32}$ A meta-analysis including patients with HTN, DM, or end-stage renal disease has shown AS, measured using PWV, as an independent predictor of total $\mathrm{CV}$ events, CV mortality and all-cause mortality. ${ }^{9}$ Additionally, studies have shown that patients with MetS, even those with 1-2 components or those without CVD or DM, have an increased risk for future CAD, CVD, all-cause mortality, and medical costs. ${ }^{7,8,14}$ In line with these reports, this study showed that high AS or MetS were risk factors for first hospitalization and all-cause mortality events in CAD patients. More importantly, we additionally found that $\mathrm{CAD}$ patients with high AS had a higher risk for developing these events than those with MetS. We believe that AS may play a more important role than MetS for worse prognosis prediction in CAD patients. However, these results need further investigations for confirmation.

A limitation of the present study was that it was conducted with a limited number of CAD patients at a single center and not all risk factors for AS development, such as smoking, were included. Therefore, more CAD patients evaluated with a more detailed survey of risk factors were required for studying the possible predictors for AS and its role in the development of future $\mathrm{CV}$ events or mortality.

\section{Conclusion}

This study demonstrated age, DM, waist circumference, and SBP to be the independent predictors for the presence of high AS in CAD patients. Additionally, CAD patients with MetS and especially the presence of high AS exhibited a significantly higher risk for first hospitalization and all-cause mortality events.

\section{Ethics Approval And Informed Consent}

The study was approved by an independent ethics committee from the Protection of the Human Subjects 
Institutional Review Board of Tzu-Chi University and Hospital (IRB099-97), and all participants gave written informed consent according to the general recommendations of the Helsinki Declaration.

\section{Data Availability}

The datasets used and/or analysed during the current study are available from the corresponding author on reasonable request.

\section{Acknowledgments}

This study was supported by a grant from the Buddhist Tzu Chi Medical Foundation, Taiwan (TCMF-MP 107-01-01). The authors would like to thank Enago for the English language review.

\section{Disclosure}

The authors report no conflicts of interest in this work.

\section{References}

1. Trayhurn P, Wood IS. Adipokines: inflammation and the pleiotropic role of white adipose tissue. Br $J$ Nutr. 2004;92:347-355. doi:10.1079/bjn20041213

2. Rosenson RS. Assessing risk across the spectrum of patients with the metabolic syndrome. Am J Cardiol. 2005;96:8E-10E. doi:10.1016/j. amjcard.2005.05.007

3. Licata G, Tuttolomondo A, Corrao S, et al. Immunoinflammatory activation during the acute phase of lacunar and non-lacunar ischemic stroke: association with time of onset and diabetic state. Int J Immunopathol Pharmacol. 2006;19:639-646. doi:10.1177/039463200601900320

4. Tuttolomondo A, Di Sciacca R, Di Raimondo D, et al. Effects of clinical and laboratory variables and of pretreatment with cardiovascular drugs in acute ischaemic stroke: a retrospective chart review from the GIFA study. Int J Cardiol. 2011;151:318-322. doi:10.1016/j.ijcard.2010.06.005

5. Di Raimondo D, Tuttolomondo A, Buttà C, et al. Effects of ACEinhibitors and angiotensin receptor blockers on inflammation. Curr Pharm Des. 2012;18:4385-4413. doi:10.2174/138161212802481282

6. Sattar N, Gaw A, Scherbakova O, et al. Metabolic syndrome with and without C-reactive protein as a predictor of coronary heart disease and diabetes in the West of Scotland Coronary Prevention Study. Circulation. 2003;108:414 419. doi:10.1161/01.CIR.0000080897.52664.94

7. Lakka HM, Laaksonen DE, Lakka TA, et al. The metabolic syndrome and total and cardiovascular disease mortality in middle-aged men. JAMA. 2002;288:2709-2716. doi:10.1001/jama.288.21.2709

8. Malik S, Wong ND, Franklin SS, et al. Impact of the metabolic syndrome on mortality from coronary heart disease, cardiovascular disease, and all causes in United States adults. Circulation. 2004;110:1245-1250. doi:10.1161/01.CIR.0000140677.20606.0E

9. Vlachopoulos C, Aznaouridis K, Stefanadis C. Prediction of cardiovascular events and all-cause mortality with arterial stiffness: a systematic review and meta-analysis. J Am Coll Cardiol. 2010;55:13181327. doi:10.1016/j.jacc.2009.10.061

10. Nam HJ, Jung IH, Kim J, et al. Association between brachial-ankle pulse wave velocity and occult coronary artery disease detected by multi-detector computed tomography. Int J Cardiol. 2012;157:227232. doi:10.1016/j.ijcard.2011.01.045
11. Kullo IJ, Bielak LF, Turner ST, Sheedy PF, Peyser PA. Aortic pulse wave velocity is associated with the presence and quantity of coronary artery calcium: a community-based study. Hypertension. 2006;47:174-179. doi:10.1161/01.HYP.0000199605.35173.14

12. Gong J, Xie Q, Han Y, et al. Relationship between components of metabolic syndrome and arterial stiffness in Chinese hypertensives. Clin Exp Hypertens. 2019;1-7. doi:10.1080/10641963.2019.1590385

13. Levisianou D, Melidonis A, Adamopoulou E, et al. Impact of the metabolic syndrome and its components combinations on arterial stiffness in Type 2 diabetic men. Int Angiol. 2009;28:490-495.

14. Nichols GA, Moler EJ. Metabolic syndrome components are associated with future medical costs independent of cardiovascular hospitalization and incident diabetes. Metab Syndr Relat Disord. 2011;9:127-133. doi:10.1089/met.2010.0105

15. Mancia G, Fagard R, Narkiewicz K, et al. 2013 ESH/ESC Guidelines for the management of arterial hypertension: the Task Force for the management of arterial hypertension of the European Society of Hypertension $(\mathrm{ESH})$ and of the European Society of Cardiology (ESC). J Hypertens. 2013;31:1281-1357. doi:10.1097/01.hjh.0000431740.32696.cc

16. Tsai JP, Wang JH, Chen ML, et al. Association of serum leptin levels with central arterial stiffness in coronary artery disease patients. $B M C$ Cardiovasc Disord. 2016;16:80. doi:10.1186/s12872-016-0371-7

17. Chen MC, Lee CJ, Yang CF, et al. Low serum adiponectin level is associated with metabolic syndrome and is an independent marker of peripheral arterial stiffness in hypertensive patients. Diabetol Metab Syndr. 2017;9:49. doi:10.1186/s13098-017-0247-8

18. Wang JH, Lee CJ, Yang CF, et al. Serum resistin as an independent marker of aortic stiffness in patients with coronary artery disease. PLoS One. 2017;12:e0183123. doi:10.1371/journal.pone.0183123

19. Alberti KG, Zimmet P, Shaw J. Metabolic syndrome-a new worldwide definition. A consensus statement from the international diabetes federation. Diabet Med. 2006;23:469-480. doi:10.1111/j.14645491.2006.01858.x

20. Laurent S, Boutouyrie P. Arterial stiffness: a new surrogate end point for cardiovascular disease? J Nephrol. 2007;20 Suppl 12:S45-S50.

21. Palombo C, Kozakova M. Arterial stiffness, atherosclerosis and cardiovascular risk: pathophysiologic mechanisms and emerging clinical indications. Vascul Pharmacol. 2016;77:1-7. doi:10.1016/j.vph.2015.11.083

22. Moreira GC, Cipullo JP, Ciorlia LA, et al. Prevalence of metabolic syndrome: association with risk factors and cardiovascular complicationss in an urban population. PLoS One. 2014;9:e105056. doi:10.1371/ journal.pone. 0105056

23. Ramirez AJ, Christen AI, Sanchez RA. Serum uric acid elevation is associated to arterial stiffness in hypertensive patients with metabolic disturbances. Curr Hypertens Rev. 2018;14:154-160. doi:10.2174/ 1573402114666180413143312

24. Cecelja M, Chowienczyk P. Dissociation of aortic pulse wave velocity with risk factors for cardiovascular disease other than hypertension: a systematic review. Hypertension. 2009;54:1328-1336. doi:10.1161/HYPERTENSIONAHA.109.137653

25. Schram MT, Henry RM, van Dijk RA, et al. Increased central artery stiffness in impaired glucose metabolism and type 2 diabetes: the Hoorn Study. Hypertension. 2004;43:176-181. doi:10.1161/01. HYP.0000111829.46090.92

26. Agnoletti D, Mansour AS, Zhang Y, et al. Clinical interaction between diabetes duration and aortic stiffness in type 2 diabetes mellitus. J Hum Hypertens. 2017;31:189-194. doi:10.1038/jhh.2016.58

27. Brunner EJ, Shipley MJ, Ahmadi-Abhari S, et al. Adiposity, obesity, and arterial aging: longitudinal study of aortic stiffness in the Whitehall II cohort. Hypertension. 2015;66:294-300. doi:10.1161/ HYPERTENSIONAHA.115.05494

28. Yun BH, Chon SJ, Cho SH, et al. Decreased renal function is a risk factor for subclinical coronary atherosclerosis in Korean postmenopausal women. J Menopausal Med. 2016;22:167-173. doi:10.6118/jmm.201 6.22.3.167 
29. Ilyas B, Dhaun N, Markie D, et al. Renal function is associated with arterial stiffness and predicts outcome in patients with coronary artery disease. QJM. 2009;102:183-191. doi:10.1093/qjmed/hcn171

30. Ford ML, Tomlinson LA, Chapman TP, Rajkumar C, Holt SG. Aortic stiffness is independently associated with rate of renal function decline in chronic kidney disease stages 3 and 4 . Hypertension. 2010;55:1110-1115. doi:10.1161/HYPERTENSIO NAHA.109.143024

31. Karras A, Haymann JP, Bozec E, et al. Large artery stiffening and remodeling are independently associated with all-cause mortality and cardiovascular events in chronic kidney disease. Hypertension. 2012;60:14511457. doi:10.1161/HYPERTENSIONAHA.112.197210
32. Zoungas S, Cameron JD, Kerr PG, et al. Association of carotid intima-medial thickness and indices of arterial stiffness with cardiovascular disease outcomes in CKD. Am J Kidney Dis. 2007;50:622630. doi:10.1053/j.ajkd.2007.07.012

33. Sipila K, Koivistoinen T, Moilanen L, et al. Metabolic syndrome and arterial stiffness: the Health 2000 Survey. Metabolism. 2007;56:320326. doi:10.1016/j.metabol.2006.10.008

34. Yokoyama H, Kuramitsu M, Kanno S, Tada J, Yokota Y, Kamikawa F. Relationship between metabolic syndrome components and vascular properties in Japanese type 2 diabetic patients without cardiovascular disease or nephropathy. Diabetes Res Clin Pract. 2007;75:200206. doi:10.1016/j.diabres.2006.06.015

\section{Publish your work in this journal}

Diabetes, Metabolic Syndrome and Obesity: Targets and Therapy is an international, peer-reviewed open-access journal committed to the rapid publication of the latest laboratory and clinical findings in the fields of diabetes, metabolic syndrome and obesity research. Original research, review, case reports, hypothesis formation, expert opinion and commentaries are all considered for publication. The manuscript management system is completely online and includes a very quick and fair peer-review system, which is all easy to use. Visit http://www.dovepress.com/testimonials.php to read real quotes from published authors. 